\title{
Sub-Nanosecond Greater-Than-10-V Compact Tunable Pulse Generator for Low-Duty-Cycle High-Peak-Power Ultra-Wideband Applications
}

\author{
Renfeng Jin, ${ }^{1}$ Subrata Halder, ${ }^{1}$ Walter R. Curtice, ${ }^{1}$ James C. M. Hwang, ${ }^{1}$ and Choi L. Law ${ }^{2}$ \\ ${ }^{1}$ Department of Electrical and Computer Engineering, Lehigh University, Bethlehem, PA 18015, USA \\ ${ }^{2}$ Department of Electrical and Electronic Engineering, Nanyang Technology University, 50 Nanyang Avenue, Singapore 639798
}

Correspondence should be addressed to Renfeng Jin, rej205@lehigh.edu

Received 9 January 2011; Revised 22 May 2011; Accepted 8 June 2011

Academic Editor: Sheng Lyang Jang

Copyright ( 2011 Renfeng Jin et al. This is an open access article distributed under the Creative Commons Attribution License, which permits unrestricted use, distribution, and reproduction in any medium, provided the original work is properly cited.

An ultra-wideband pulse generator was designed and fabricated in GaAs HBT IC technology. The generator includes delay and differential circuits to convert a TTL input into a Gaussian pulse signal as well as a Class-C amplifier to boost the pulse amplitude while compressing the pulse width. By adjusting the collector bias of the Class-C amplifier, the pulse amplitude can be varied linearly between $3.5 \mathrm{~V}$ and $11.5 \mathrm{~V}$ while maintaining the pulse width at $0.3 \pm 0.1$ nanosecond. Alternatively, by adjusting the base bias of the Class-C amplifier, the pulse width can be varied linearly between $0.25 \mathrm{~ns}$ and $0.65 \mathrm{~ns}$ while maintaining the pulse amplitude at $10 \pm 1 \mathrm{~V}$. Finally, the amplified Gaussian signal can be shaped into a monocycle signal by an $L$ - $C$ derivative circuit. The present pulse generator compares favorably with pulse generators fabricated in CMOS ICs, step-recovery diodes, or other discrete devices.

\section{Introduction}

ULTRA-wideband (UWB) impulse radio [1] is attractive for applications such as through-wall imaging, precision navigation, location, and tracking. A UWB impulse radio can be particularly attractive for high-resolution ranging applications, if low-duty-cycle high-peak-power transmitters can be readily assembled from sub-nanosecond high-voltage pulse generators. Additionally, pulse generators capable of tunable amplitude and width can enhance the functionality of the UWB impulse radio. For example, the pulse amplitude can be adjusted according to the range of interest, while the pulse width can be varied to inspect objects at different depths inside a wall.

Sub-nanosecond high-voltage pulse generators are required by low-duty-cycle high-peak-power UWB transmitters to maximize their performance without exceeding the FCC limits of $-41.3 \mathrm{dBm} / \mathrm{MHz}$ and $0 \mathrm{dBm} / 50 \mathrm{MHz}$ for average and peak powers, respectively [2]. For pulse-repetition frequencies of $187.5 \mathrm{kHz}$ or lower, peak power instead of average power is the main limit. In this case, for a pulse width of 0.5 nanosecond, the pulse amplitude can be as high as $8.9 \mathrm{~V}$ on a $50-\Omega$ load [3]. Considering connector loss, antenna mismatch, and so forth, sub-nanosecond greaterthan-10-V pulse generators are required.

To satisfy the aforementioned requirement, we designed [4] a sub-nanosecond greater-than- $10-\mathrm{V}$ tunable pulse generator by using the GaAs HBT IC technology. As shown in Figure 1 and Table 1, this pulse generator could generate much higher pulse amplitude than pulse generators based on Si CMOS or BiCMOS ICs. It was also much more compact than pulsed generators based on step-recovery diodes (SRDs) and other discrete devices. This paper expands on [4] mainly by discussing in detail the device modeling, circuit design, simulation, and testing of the present pulse generator. Additionally, modeling, simulation, and testing of the temperature stability of the pulse generator are presented, stability over four orders of pulse-repetition frequencies is characterized at different temperatures, output power 
TABLE 1: Performance of UWB pulse generators.

\begin{tabular}{|c|c|c|c|c|c|c|c|c|c|}
\hline Technology & Waveform & $\begin{array}{c}\text { Pulse } \\
\text { amplitude } \\
\text { (V) }\end{array}$ & $\begin{array}{c}\text { Pulse } \\
\text { width } \\
\text { (nanosecond) }\end{array}$ & $\begin{array}{l}\text { Power } \\
\text { cons. } \\
(\mathrm{mW})\end{array}$ & $\begin{array}{l}\text { Supply } \\
\text { voltage } \\
\text { (V) }\end{array}$ & $\begin{array}{c}\text { Die } \\
\text { size } \\
\left(\mathrm{mm}^{2}\right) \\
\end{array}$ & $\begin{array}{l}\text { Eff. } \\
(\%)\end{array}$ & $\begin{array}{c}\text { Jitter } \\
\text { (ps) }\end{array}$ & Ref. \\
\hline \multirow[t]{2}{*}{ GaAs HBT } & Impulse & $3.5-11.5$ & $0.25-0.65$ & \multirow{2}{*}{120} & \multirow{2}{*}{$3.3-14$} & \multirow{2}{*}{$0.95 \times 0.95$} & - & \multirow[t]{2}{*}{$<1.5$} & \multirow[t]{2}{*}[4]{} \\
\hline & Monocycle & $5.1-8.8$ & $0.2-1.0$ & & & & 0.8 & & \\
\hline GaAs HEMT & Impulse & 0.7 & 0.06 & 120 & - & $1.5 \times 1.2$ & 一 & $0.5-1.5$ & [11] \\
\hline \multirow{2}{*}{ Si SRD } & Monocycle & 8 & $0.6-1.1$ & - & 18 & $40 \times 60$ & - & & [12] \\
\hline & Impulse & 4.35 & 0.25 & - & 15 & $60 \times 60$ & - & - & [13] \\
\hline Si BJT & Monocycle & 1.3 & 0.5 & - & - & - & - & - & [14] \\
\hline \multirow{8}{*}{ Si MOSFET } & Impulse & 1.4 & 0.4 & - & 2.5 & $0.3 \times 0.25$ & - & - & [15] \\
\hline & Monocycle & $0.3-0.6$ & $0.14-0.35$ & - & & & - & & \\
\hline & Impulse & 2.8 & 0.5 & - & 3.3 & 0.5 & - & - & [16] \\
\hline & Impulse & 1.2 & 2.0 & 0.72 & 1.8 & $1.71 \times 0.71$ & - & - & [17] \\
\hline & Monocycle & 3.7 & 0.5 & - & - & - & - & - & [18] \\
\hline & Impulse & $0.03-0.12$ & $0.07-0.18$ & 17 & 1 & 0.0068 & - & - & [19] \\
\hline & Monocycle & 0.66 & 0.38 & 19.8 & 1.2 & 0.25 & 2.6 & - & [20] \\
\hline & Monocycle & 0.175 & 0.3 & 0.18 & 1.8 & $\begin{array}{c}0.175 \times \\
0.175\end{array}$ & - & - & [21] \\
\hline
\end{tabular}

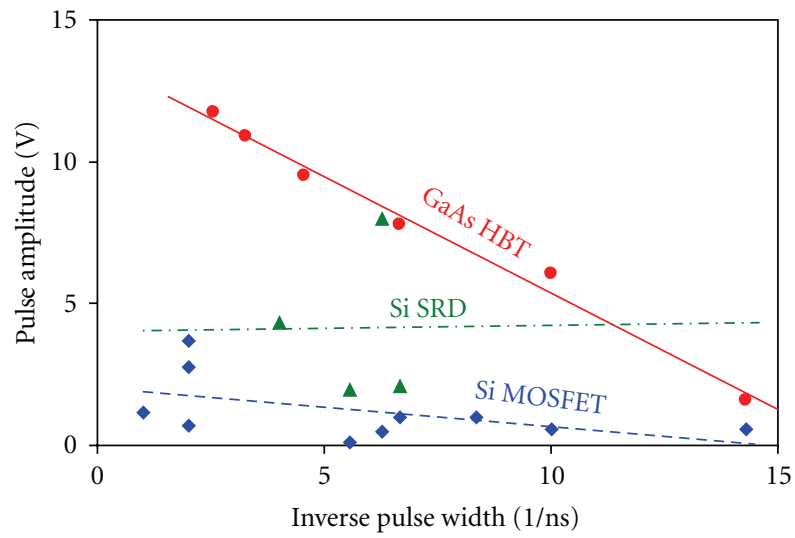

FIGURE 1: Output pulse amplitude as a function of inverse pulse width for UWB pulse generators of different technologies.

spectrum is compared with the FCC mask, and the effect of load impedance is modeled and measured.

\section{Device Technology and Modeling}

The GaAs HBT IC technology is chosen for not only its superior combination of high-voltage and high-frequency characteristics to that of Si CMOS or BiCMOS IC technology but also its much enhanced output power capacity for low-duty-cycle isothermal operation [5], which helps compact the size of the pulse generator. Because the thermal conductivity of GaAs is three times lower than that of $\mathrm{Si}$, ordinarily, the power capacity of GaAs HBTs is limited by thermally induced current collapse [6]. However, this is not an issue under low-duty-cycle isothermal operation. So the traditional design approach is not suitable to GaAs HBT

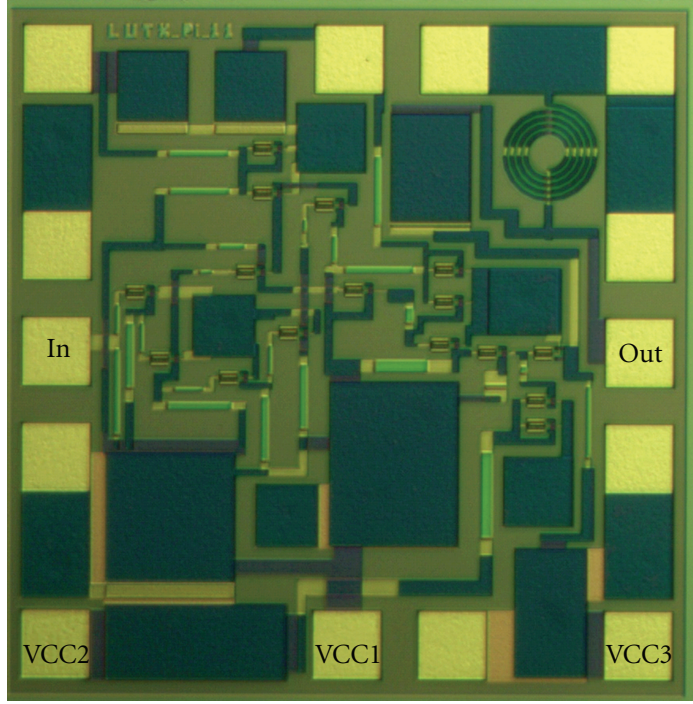

Figure 2: Micrograph of the $\sim 1 \mathrm{~mm}^{2}$ GaAs HBT IC pulse generator.

pulse generator. In our design, a Darlington pair is used to keep the main amplifier at the same temperature, which greatly enhances the output power of the pulse generator. Used in most mobile phones, the GaAs HBT technology is also relatively mature and low cost. In comparison, GaAs HEMTs often suffer from gate lag while GaN HEMTs are less mature.

Figure 2 shows the tunable pulse generator fabricated by a commercial GaAs HBT foundry [7]. The die size is less than $1 \mathrm{~mm} \times 1 \mathrm{~mm}$, which includes not only all HBTs but also all bias resistors, capacitors, and DC/RF probe pads. To ensure compact size and wideband performance, the pulse generator 


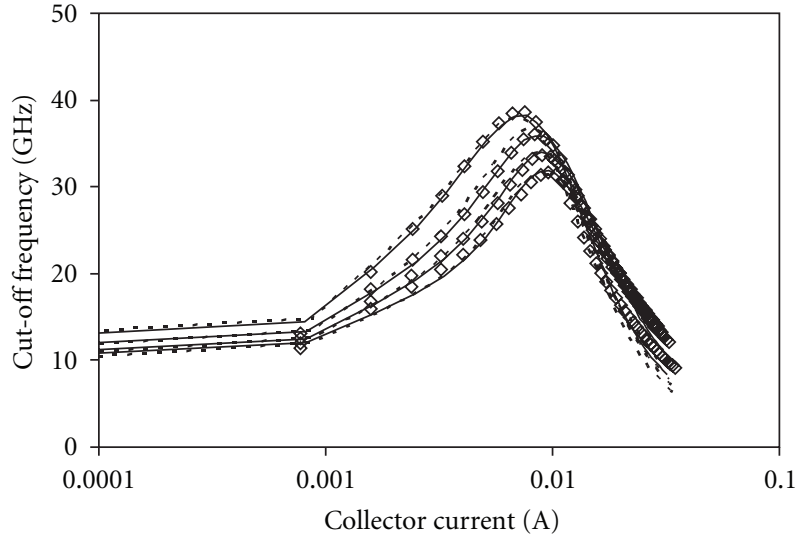

(a)

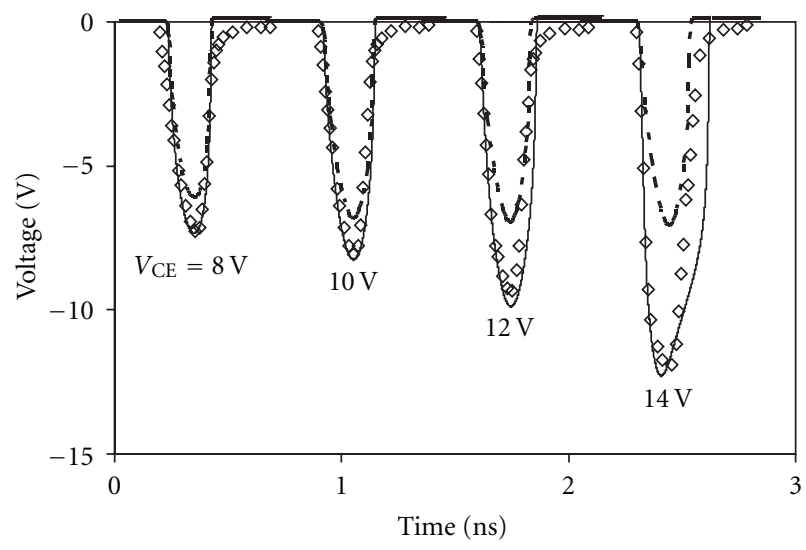

(b)

Figure 3: Measured (symbol) (a) cutoff frequency $f_{T}$ and (b) impulse response of a typical HBT versus that simulated by using the Agilent (dashed curve) and modified (solid curve) HBT model. In (a), $V_{\mathrm{CE}}=1,2,3$, and $4 \mathrm{~V}$ top down. In (b), the input pulse width and amplitude are 0.2 nanosecond and $1.45 \mathrm{~V}$, respectively. $V_{\mathrm{BE}}=0$. Artificial delays between impulses are added for clarity.

contains only one small inductor, which is part of the L-C derivative circuit. Extra probe pads are included for diagnosis but are not required for establishing the circuit performance. The die size could have been at least halved but was laid out to match the footprint of other circuits.

Typically, the HBTs have a current-gain cutoff frequency $f_{T}$ of $40 \mathrm{GHz}$, a maximum frequency of oscillation $f_{\mathrm{MAX}}$ of $60 \mathrm{GHz}$, a common-emitter breakdown voltage $\mathrm{BV}_{\mathrm{CEO}}$ of $15 \mathrm{~V}$, and a common-base breakdown voltage $\mathrm{BV}_{\mathrm{CBO}}$ of $30 \mathrm{~V}$. For accurate simulation of sub-nanosecond high-voltage operation, a modified HBT model $[8,9]$ was extracted and then implemented in the ADS [10] circuit design environment.

Power is the product of current and voltage. Ordinarily, the HBT current is limited by the Kirk (base push-out) effect, which is highly temperature-dependent, while its voltage is limited by thermal runaway [6]. Under isothermal operation, we found that the HBT current and voltage capacities could be greatly extended, resulting in much higher power output. In this case, the Kirk effect is not only current-dependent but also voltage-dependent, and the maximum voltage is limited by avalanche breakdown instead of thermal runaway. However, since the commercially available Agilent HBT model [10] includes limited Kirk effect and no breakdown, we modified it by adding stronger voltage dependence to the conventional Kirk model and current dependence to the conventional breakdown model.

Specifically, the Kirk charge $Q_{K}$ in the Agilent HBT model is replaced with the following:

$$
Q_{K}=\frac{I_{F} W_{\mathrm{CIB}}^{2}}{4 D_{\mathrm{NC}}}
$$

where $I_{F}$ is the forward current from the collector to the base, $W_{\mathrm{CIB}}$ is the extended width of the base, and $D_{\mathrm{NC}}$ is the electron diffusivity in the extended base. $W_{\mathrm{CIB}}$ is defined as

$$
\begin{aligned}
& W_{\mathrm{CIB}}=W_{C}-\sqrt{\frac{2 \varepsilon V_{\mathrm{CBK}}}{\left(I_{F} / A_{E} v_{\mathrm{SAT}}-q N_{C}\right)}} ; \\
& \frac{1}{v_{\mathrm{SAT}}}=\frac{1}{v_{\mathrm{SAT} 0}}+k \Delta T,
\end{aligned}
$$

where $W_{C}$ is the collector width, $\varepsilon$ is the GaAs permittivity, $A_{E}$ is the emitter area, $q$ is the electron charge, $N_{C}$ is the collector doping, $\Delta T$ is the difference between junction temperature $T$ and room temperature $T_{0}, v_{\mathrm{SAT}}$ and $v_{\mathrm{SAT} 0}$ are saturated electron velocities at $T$ and $T_{0}$, respectively, and $k$ is a fitting parameter. Equation (2) follows the conventional form [22] except that $V_{\mathrm{CBK}}$ is dependent on $V_{\mathrm{CB}}+V_{\mathrm{BI}}$ superlinearly:

$$
V_{\mathrm{CBK}}=\left(V_{\mathrm{CB}}+V_{\mathrm{BI}}\right)\left\{1+\left[\frac{\left(V_{\mathrm{CB}}+V_{\mathrm{BI}}\right)}{V_{\mathrm{CK}}}\right]^{\mathrm{CK}}\right\},
$$

where $V_{\mathrm{CB}}$ and $V_{\mathrm{BI}}$ are the bias and built-in voltages of the collector-base junction, and $V_{\mathrm{CK}}$ and $\mathrm{CK}$ are fitting parameters. Meanwhile, $D_{\mathrm{NC}}$ is also made current-dependent:

$$
D_{\mathrm{NC}}=D_{\mathrm{NC} 0}\left(\frac{I_{C}}{I_{\mathrm{KCR}}}\right)^{-\mathrm{GK}}\left(\frac{T}{T_{0}}\right),
$$

where $D_{\mathrm{NC} 0}$ is the low-field electron diffusivity in the collector and $I_{\mathrm{KCR}}$ and GK are fitting parameters. Finally, as in the Agilent HBT model, $Q_{K}$ is partitioned between the collector and base:

$$
Q_{\mathrm{CK}}=F_{\mathrm{EXKE}} Q_{K}, \quad Q_{\mathrm{BK}}=\left(1-F_{\mathrm{EXKE}}\right) Q_{K},
$$

where $F_{\text {EXKE }}$ is yet another fitting parameter.

Current dependence is added to the breakdown model through an additional hyper-cosine term in the multiplication factor $M$ while temperature dependence is captured by a power term:

$$
\begin{gathered}
M=1+m \cosh \left[\left(I_{F}-A_{E} N_{C} q v_{\mathrm{SAT}}\right) V_{\mathrm{CB}}\right] \\
\tan \left[\left(\frac{\pi}{2}\right)\left(\frac{V_{\mathrm{CB}}}{\mathrm{BV}_{\mathrm{CBO}}}\right)^{n}\right]\left(\frac{T}{T_{0}}\right)^{-X M},
\end{gathered}
$$

where $m, n$, and $X M$ are fitting parameters.

To properly account for the transport across the HBT collector, self-consistent solutions are usually obtained for 


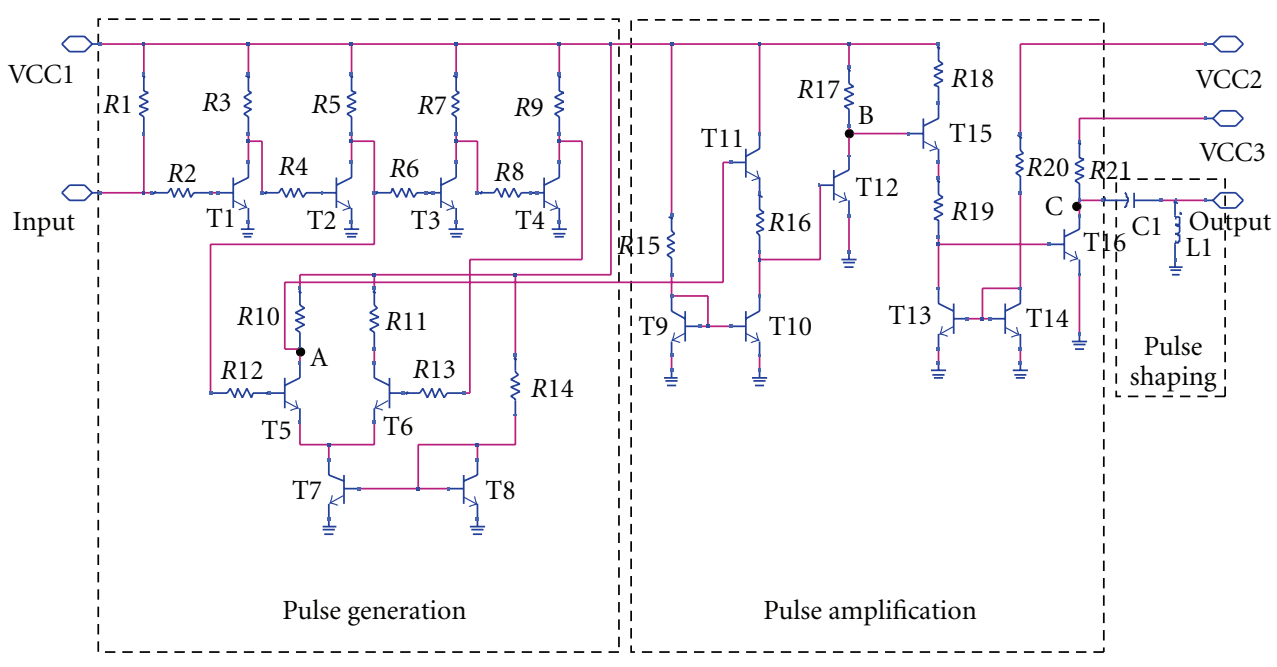

FIGURE 4: Circuit schematics showing the pulse generator comprises the pulse generation, amplification, and shaping blocks.

the Poisson and drift-diffusion equations, which are coupled through the electric field. Under low currents, abruptdepletion and quasineutral approximations apply; so the field varies linearly in the depletion region and is nil in the undepleted collector. However, under high currents, space charge density varies significantly in the collector and the field distribution becomes highly nonlinear. For example, the collector can be totally depleted and the field can even be reversed. Under such complicated field distribution, the numerical solutions are too complicated for compact modeling [5]. Therefore, instead of exact solutions, we empirically added the current dependence in (6) to account for the high-current effect on the field distribution and, in turn, the multiplication factor. Similarly, when the field distribution in the collector becomes very complicated, so does the electron velocity in the collector. However, we retained the constant $v_{\mathrm{SAT}}$ in (2) and (6) and approximated the effect of velocity variation across the collector with additional voltage and current dependence in (3), (4), and (6). In this case, " $v_{\mathrm{SAT}}$ " represents some weighted average of the velocity variation and may deviate from the commonly assumed value of $10^{7} \mathrm{~cm} / \mathrm{s}$.

With proper account of the Kirk and breakdown effects under high-voltage high-current operations, the collector current $I_{C}$ can be modeled as

$$
I_{C}=I_{\mathrm{BC}}+\frac{d\left(Q_{\mathrm{TB}}+Q_{\mathrm{TC}}+Q_{\mathrm{CK}}\right)}{d t}+M I_{F},
$$

where the base-collector diode current $I_{\mathrm{BC}}$ and the base and collector delay charges $Q_{\mathrm{TB}}$ and $Q_{\mathrm{TC}}$ are from the original Agilent HBT model.

The above-modified HBT model was coded in VerilogA [23] and installed in ADS [10] to aid the circuit design. Figure 3 shows that while both the Agilent and the modified models can fit the small-signal HBT characteristics, the modified model is superior to the Agilent model in predicting the HBT impulse response. Therefore, the modified HBT model is used in the design of pulse generators as described in the following.

\section{Circuit Design}

Figure 4 shows schematically the circuit design of the pulsegeneration, pulse-amplification, and pulse-shaping blocks of the pulse generator. The pulse-generation block includes a delay chain of HBTs $T 1, T 2, T 3$, and $T 4$ and a differential amplifier of HBTs $T 5$ and $T 6 . T 5$ and $T 6$ are driven by $T 2$ and $T 4$, respectively. The delay time $\tau$ between $T 2$ and $T 4$ is dominated by the $R-C$ time constant of the load resistance on the collector of each HBT and the load capacitance between the collector of one HBT and the base of the next HBT. For the present HBTs, $R \approx 1000 \Omega$ and $C \approx 0.2 \mathrm{pF}$. Therefore, $\tau \approx 2 R C \approx 0.4$ nanosecond and the pulse-generation block can generate sub-nanosecond positive and negative pulses from the falling and rising edges, respectively, of the TTL input (Figure 5). The input amplitude to $T 6$ is higher than that of $T 5$ because the base current of $T 3$ introduces additional voltage drop over $R 5$. Once $T 6$ is conducting, the output of $T 5$ is clamped at the high voltage whether $T 5$ is conducting or not. This helps maximize the single-ended output of the differential amplifier T5-T6 while suppressing the generation of positive pulses. At the ensuing Class-C pulse-amplification block, the relatively strong negative pulses are further amplified and sharpened while the relatively weak positive pulses are further suppressed.

The pulse-amplification block includes two Darlington pairs, $T 11-T 12$ and $T 15-T 16$, respectively. $T 12$ is biased in the saturation region so that the first Darlington pair serves as the driver amplifier; $T 16$ is biased in the cutoff region so that the second Darlington pair acts as a Class-C amplifier. The Class-C bias of $T 16$ helps ensure isothermal operation, minimize power consumption, cut off low-voltage ringing, compress pulse width, and prevent oscillation. Current mirrors T9-T10 and T13-T14 limit the currents through T11 and $T 15$, respectively. The pulse-shaping block is a si-mple high-pass $L-C$ derivative circuit. If necessary, higher-order derivative circuits can be added to shape the pulse further and to take advantage of the full bandwidth of 3.1-10.6 GHz. 
All HBTs in the pulse generator are of the same design with an emitter area of $2 \mu \mathrm{m} \times 20 \mu \mathrm{m}$, except that $T 16$ has an emitter area of $2 \mu \mathrm{m} \times 20 \mu \mathrm{m} \times 4$. The collector of T16 is shunted to VCC 3 through a $1 \mathrm{k} \Omega$ resistor, which provides adequate $\mathrm{DC}-\mathrm{RF}$ isolation and helps reduce ringing. Although this resistor consumes little power as T16 is normally off, it cannot be made much bigger without degrading the performance at high pulse-repetition frequencies. When $T 16$ is turned on by the input pulse, the output impedance of $T 16$ quickly approaches $50 \Omega$, as evidenced by the absence of ringing or other delayed reflections in both simulation and measurement. This large-signal transient impedance can be adjusted by varying the size and bias of $T 16$ to better suit that of the antenna, especially UWB antennas with higher-than$50-\Omega$ impedances.

The output pulse width can be tuned by adjusting VCC2, which affects the base bias of $T 16$ through $R 19$. The output pulse amplitude can be tuned by adjusting VCC3, which affects the collector bias of T16 through $R 21$. Usually, the output pulse amplitude of T16 may be limited by both self-heating and avalanche breakdown. For the present lowduty-cycle sub-nanosecond pulse generator, self-heating is not a concern because the HBT thermal time constant is on the order of microsecond [5]. Avalanche breakdown is suppressed by adding R19 and T14 to the base of T16 [24]. Typically, VCC1 $=3.3 \mathrm{~V}, \mathrm{VCC} 2=2-5 \mathrm{~V}$, and VCC3 $=4-14 \mathrm{~V}$.

Figure 5 shows the simulated voltage waveforms at the input and output of the pulse generator, as well as the internal nodes A, B, and C labeled on Figure 4. It can be seen that at Node A, negative pulses are generated from the rising edge of a $10 \mathrm{MHz}$ square-wave input signal, while positive pulses generated from the falling edge of the input are barely discernable. The negative pulses are then inverted and amplified once at Node B and twice at Node C, while the positive pulses are completely suppressed. Finally, the output signal becomes monocycle after going through the $L-C$ pulseshaping block.

\section{Results and Discussion}

The fabricated pulse generators were tested by using a previously described setup [8] with the TTL input generated by an HP 8116A function generator. The output waveforms were sampled by an Agilent 86100 oscilloscope and deembedded to the die input and output pads after accounting for the frequency response of the cable assemblies. Unless otherwise noted, most pulse generators were tested with a $0.5-2.5-\mathrm{V}$ TTL square signal of $10 \mathrm{MHz}$ pulse-repetition frequency (Figure 5), which corresponds to $<1 \%$ duty cycle for the submicron pulses.

As predicted by simulation, Figure 6 shows that the pulse amplitude at Node $\mathrm{C}$ of the pulse generator can be tuned linearly between $3.5 \mathrm{~V}$ and $11.5 \mathrm{~V}$ by varying VCC3 from $4 \mathrm{~V}$ to $14 \mathrm{~V}$, while maintaining the pulse width within 0.3 \pm 0.1 nanosecond. (In this paper, the pulse amplitude is meas-ured peak-to-peak, while the pulse width is the full width at half maximum.) Figure 7 shows that the pulse width at Node $\mathrm{C}$ can be tuned linearly between 0.25 nanosecond and 0.65 nanosecond by varying VCC2 from $2.5 \mathrm{~V}$ to $4.5 \mathrm{~V}$,

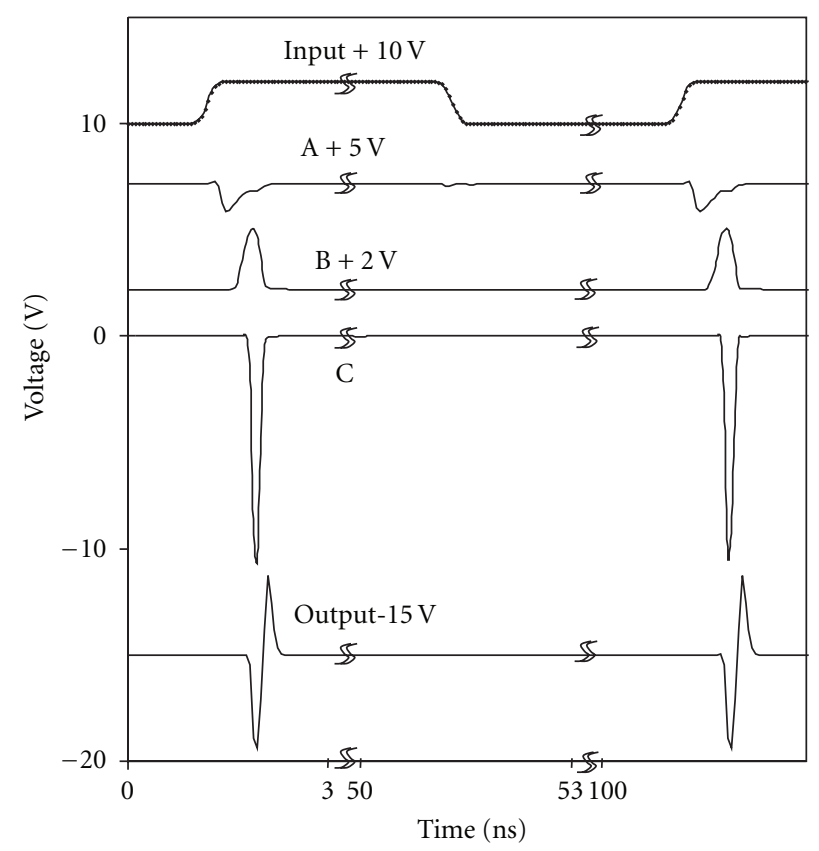

FIGURE 5: Simulated voltage waveforms at the input and output of the pulse generator as well as the internal nodes A, B, and C labeled in Figure 4. The negative pulse generated from the rising edge of the input signal is progressively amplified and shaped, while the positive pulse generated from the falling edge of the input signal is barely discernable at A and is completely suppressed at the output. Artificial offset voltages are added for clarity.

while maintaining the pulse amplitude at $10 \pm 1 \mathrm{~V}$. In comparison, VCC1 could be varied from $3.3 \mathrm{~V}$ to $3.6 \mathrm{~V}$ without obvious impact on output pulse amplitude or width. Similarly, no obvious jitter was observed on the oscilloscope with 1.5-ps time resolution. Both Figures 6 and 7 include additional temperature-dependent data. Without adjusting the bias or input conditions, the pulse generator was found to operate similarly well when the ambient temperature was varied from $-40^{\circ} \mathrm{C}$ to $85^{\circ} \mathrm{C}$. Figure $6(\mathrm{~b})$ shows that the pulse amplitude at Node $\mathrm{C}$ varies by less than $0.2 \mathrm{~V}$ across the temperature range measured, except at the highest VCC3 when it is limited by the Kirk threshold that decreases with increasing temperature. On the other hand, as shown in Figure 7(b), the pulse width increases with increasing temperature at all VCC2 values, because the high-frequency gain decreases with increasing temperature. To further improve the temperature performance of the pulse generator, temperature sensing and compensating circuit can be incorporated to fine-tune VCC2.

Following the pulse-shaping block, the pulses at Node C are converted to monocycles at the output. Figure 8 shows that with VCC2 varying between $2 \mathrm{~V}$ and $6 \mathrm{~V}$, the monocycle amplitude varies from $5.1 \mathrm{~V}$ to $8.8 \mathrm{~V}$ while its width varies from 0.2 nanosecond to 1.0 nanosecond. The positive and negative portions of the monocycle differ mainly due to the low-quality factor of the inductor. Figure 9 shows the corresponding power spectrum for monocycles generated with VCC1 $=3.3 \mathrm{~V}, \mathrm{VCC} 2=6 \mathrm{~V}$, and VCC3 $=13 \mathrm{~V}$ under a pulse-repetition frequency of $3 \mathrm{MHz}$. Obviously, a 


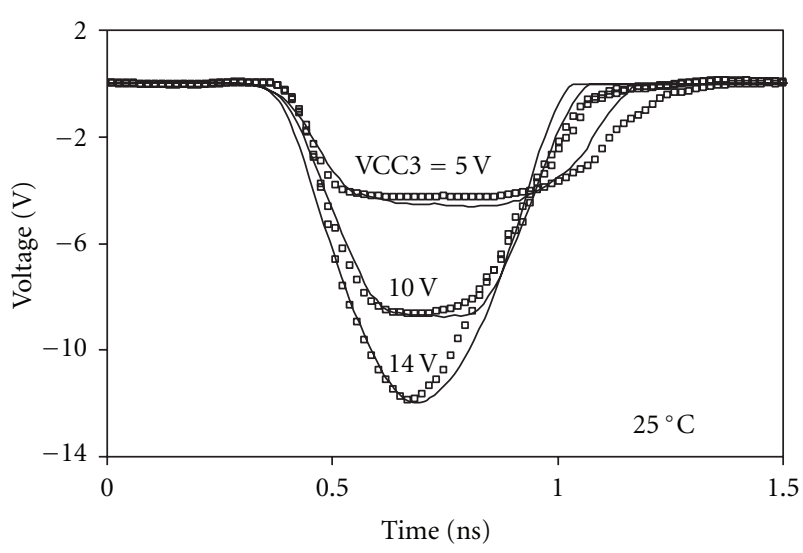

(a)

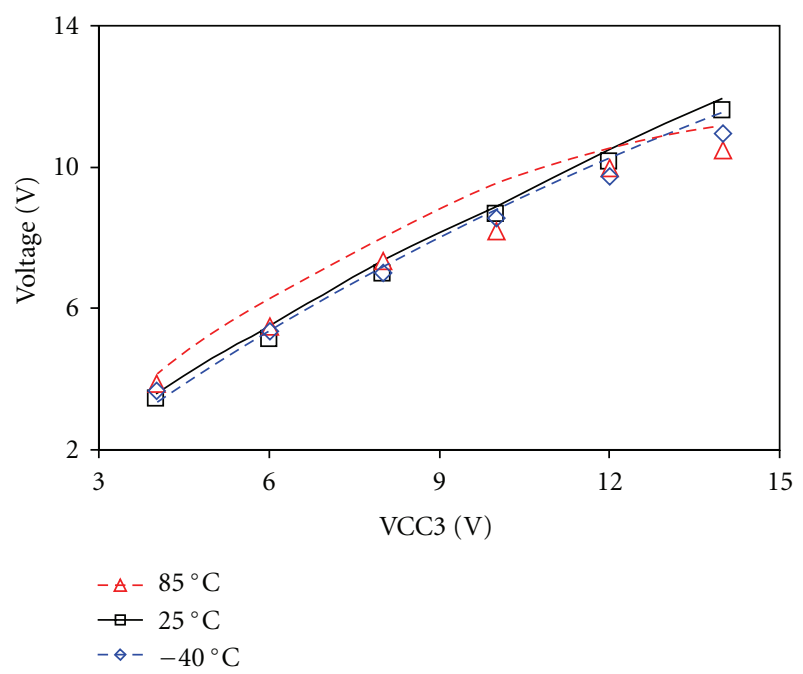

(b)

Figure 6: Measured (symbol) versus simulated (curve) (a) waveform and (b) pulse amplitude at Node $\mathrm{C}$ of the pulse generator with $\mathrm{VCC} 1=\mathrm{VCC} 2=3.3 \mathrm{~V}$, and $\mathrm{VCC} 3=4-14 \mathrm{~V}$.

notch filter around $2 \mathrm{GH}$ is needed to prevent interference with global positioning systems, cellular communications, and industrial, scientific, and medical instrumentations. On the other hand, the power density elsewhere can be boosted by increasing the pulse-repetition frequency and the entire spectrum can be shifted to higher frequencies by incorporating higher-order derivative circuits. Figure 10 shows that the output amplitude of the pulse generator is stable under different pulse-repetition frequencies between $40 \mathrm{KHz}$ and $25 \mathrm{MHz}$, which indicates that the isothermal approximation is valid over a wide range of pulse amplitudes and duty cycles.

High impedance antennas are often used in UWB systems; so the pulse generator was evaluated by increasing the load impedance from $50 \Omega$ to $200 \Omega$. No oscillation was observed. Figure 11 shows that under a constant input voltage of $2 \mathrm{~V}$ and a load impedance of $200 \Omega$, the pulse amplitude at Node C is slightly lower than VCC3 by the HBT knee voltage of approximately $1 \mathrm{~V}$. However, at lower load

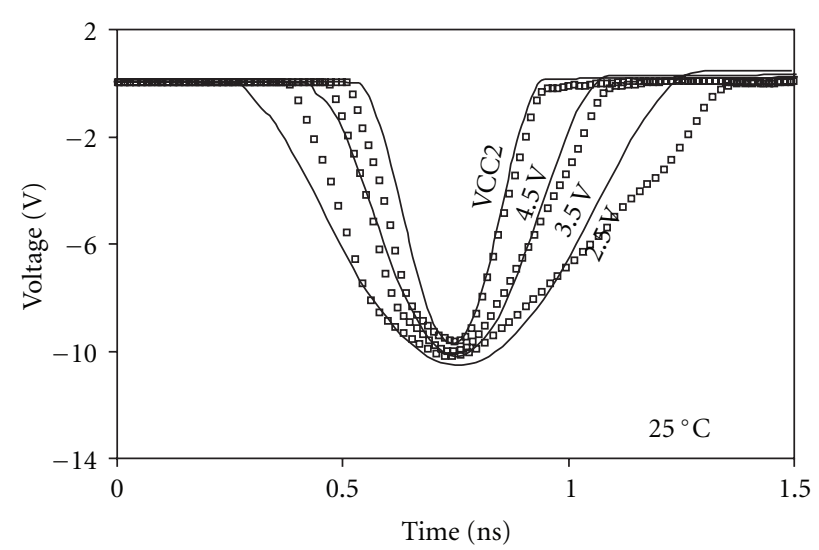

(a)

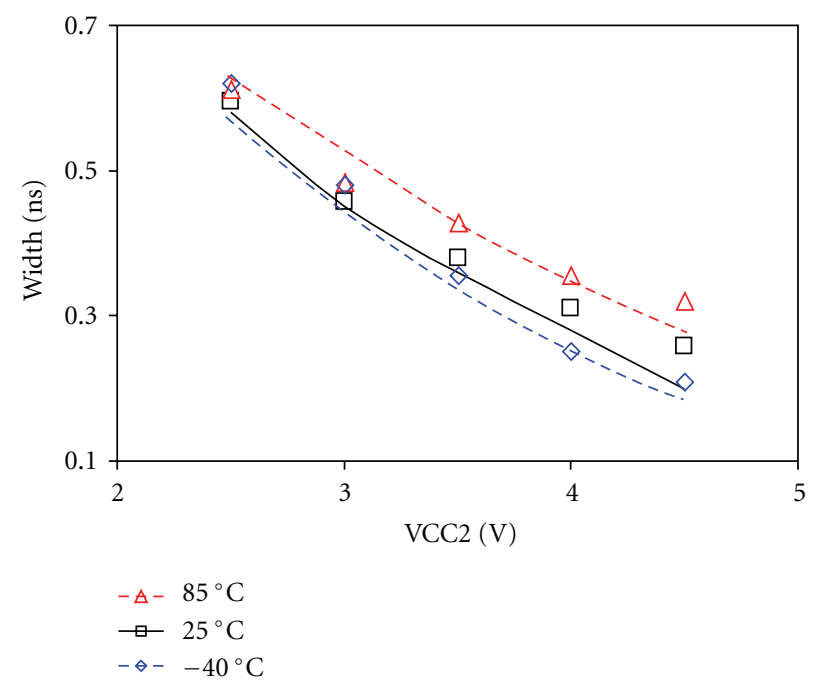

(b)

Figure 7: Measured (symbol) versus simulated (curve) (a) waveform and (b) width of impulses at Node $\mathrm{C}$ of the pulse generator with $\mathrm{VCC} 1=3.3 \mathrm{~V}, \mathrm{VCC} 2=2.5-4.5 \mathrm{~V}$, and $\mathrm{VCC} 3=12 \mathrm{~V}$.

impedances such as $100 \Omega$ and $50 \Omega$, the pulse amplitude saturates at a value much lower than VCC3 unless the input voltage is significantly increased to overdrive the pulse generator. In this case, although the pulse generator could output $12 \mathrm{~V}$ into different load impedances, the output power would decrease with increasing load impedance. However, if both the input and bias conditions could be fine-tuned at each impedance, then the minimum pulse width would decrease with increasing load impedance, too. For example, after such fine tuning, the minimal pulse widths with $10 \mathrm{~V}$ pulse amplitude are 0.25 nanosecond, 0.21 nanosecond, and 0.20 nanosecond for $50 \Omega, 100 \Omega$, and $200 \Omega$ loads, respectively.

The present pulse generator consumes approximately $120 \mathrm{~mW}$, with $100 \mathrm{~mW}$ flowing through the pulse-amplification block. As listed in Table 1, the ratio of power consumption over pulse amplitude for the present pulse generator is comparable to that of the pulsed generators made of GaAs HEMTs and Si MOSFETs. The power consumption 


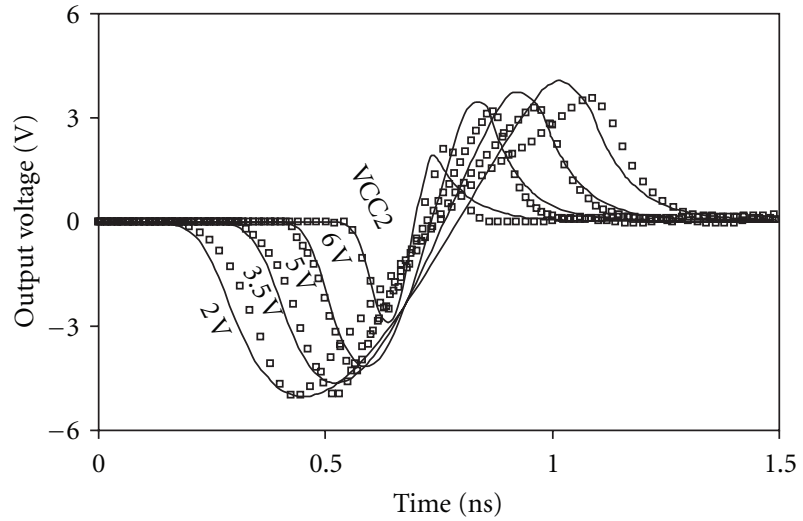

Figure 8: Measured (symbol) versus simulated (curve) monocycles generated with VCC1 $=3.3 \mathrm{~V}, \mathrm{VCC} 2=2-6 \mathrm{~V}$, and VCC3 $=13 \mathrm{~V}$.

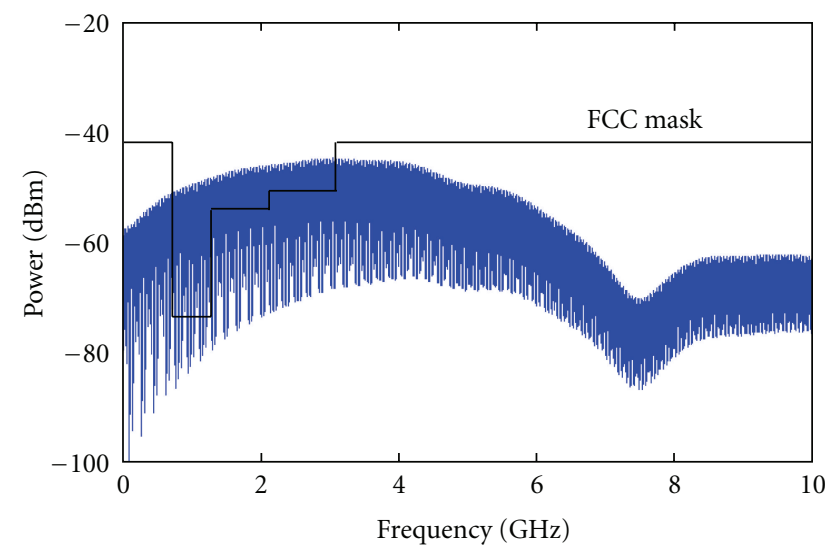

Figure 9: Power spectrum of monocycles generated with VCC1 = $3.3 \mathrm{~V}, \mathrm{VCC} 2=6 \mathrm{~V}, \mathrm{VCC} 3=13 \mathrm{~V}$, and $\mathrm{PRF}=3 \mathrm{MHz}$.

of the present pulse generator can be reduced by reducing the size of certain HBTs. For example, reducing T1-T8, T9, and $T 14$ from $40 \mu \mathrm{m}^{2}$ to $8 \mu \mathrm{m}^{2}$ would save $70 \%$ of power. Much greater power can be saved by cycling off the pulse amplifier when no pulse is expected. With an on-time of 1 nanosecond and a pulse-repetition frequency of $10 \mathrm{MHz}$, the power consumed by the pulse amplifier can be reduced by a factor of 100 to approximately $1 \mathrm{~mW}$. The power saving can be even greater at lower pulse-repetition frequencies. At approximately $1 \mathrm{~mm}^{2}$, the die costs less than $\$ 1$ for volume production.

\section{Conclusion}

For low-duty-cycle high-peak-power ultra-wideband applications, a sub-nanosecond greater-than- $10-\mathrm{V}$ tunable pulse generator was designed by taking advantage of the recent discovery of much greater output capacity of GaAs HBTs under sub-nanosecond isothermal operation. To aid the circuit design, a commercially available HBT model was modified by adding stronger voltage dependence to the Kirk-effect model and current dependence to avalanche-breakdown model. Using the modified HBT model, the performance of the

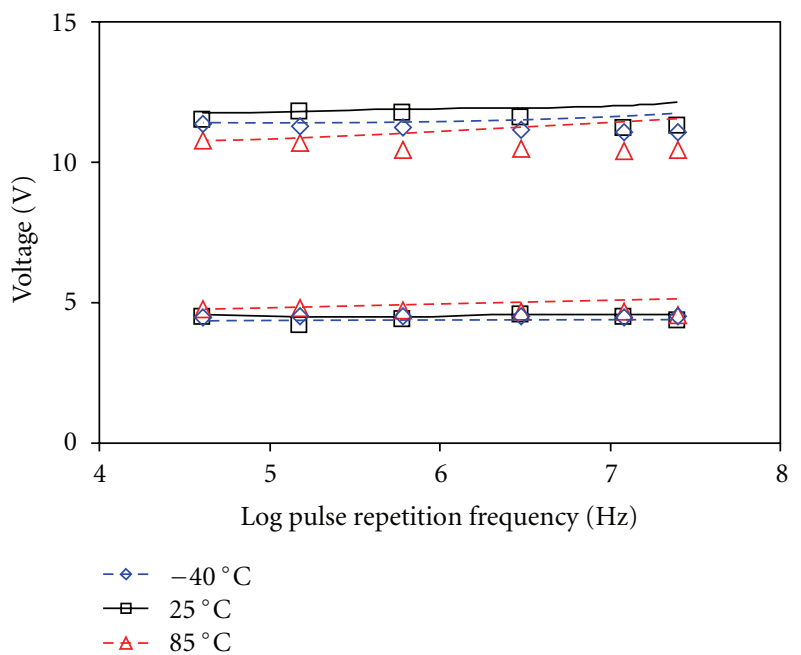

FIGURE 10: Measured output pulse amplitudes under different temperature as functions of pulse-repetition frequency between $40 \mathrm{kHz}$ and $25 \mathrm{MHz}$. From the bottom up, the biases for pulse generator are $\mathrm{VCC} 1=3.3 \mathrm{~V}, \mathrm{VCC} 2=3.3 \mathrm{~V}$, and $\mathrm{VCC} 3=14 \mathrm{~V}$ and $\mathrm{VCC} 1=3.3 \mathrm{~V}, \mathrm{VCC} 2=3.3 \mathrm{~V}$, and $\mathrm{VCC} 3=6 \mathrm{~V}$.

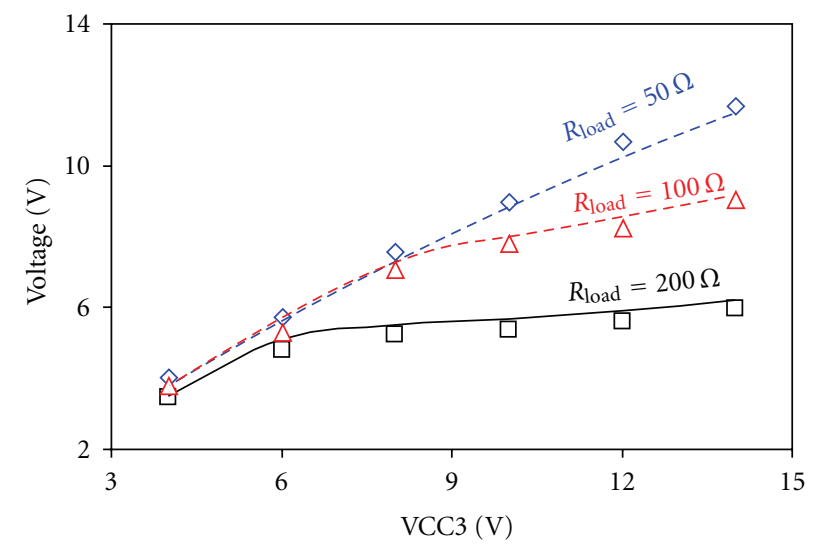

FIGURE 11: Measured (symbol) versus simulated (curve) amplitude of impulses generated with $\mathrm{VCC} 1=3.3 \mathrm{~V}, \mathrm{VCC} 2=6.5 \mathrm{~V}$, and VCC3 $=4-14 \mathrm{~V}$.

fabricated pulse generators was accurately predicted. For example, the output pulse amplitude could be varied linearly between $3.5 \mathrm{~V}$ and $11.5 \mathrm{~V}$ while maintaining the pulse width at $0.3 \pm 0.1$ nanosecond. Alternatively, the pulse width could be varied linearly between 0.25 nanosecond and 0.65 nanosecond while maintaining the pulse amplitude at $10 \pm 1 \mathrm{~V}$. Without adjusting the bias or input conditions, this performance was found to vary little when the ambient temperature was varied from $-40^{\circ} \mathrm{C}$ to $85^{\circ} \mathrm{C}$. These results show that the present pulse generator has much higher output capacity than those fabricated in CMOS ICs are and is much more compact than those fabricated in discrete devices are.

\section{Acknowledgment}

This work was supported in part by Commonwealth of Pennsylvania, Department of Community and Economy 
Development, under Pennsylvania Infrastructure Technology Alliance (PITA).

\section{References}

[1] R. Aiello and A. Batra, Ultra Wideband Systems: Technologies and Applications, Elsevier, Boston, Mass, USA, 2006.

[2] US Federal Communications Commission, "Revision of part 15 of the commission's rules regarding ultra-wideband transmission system: first report and order," Tech. Rep. 98-153, ET Docket, 2002, FCC 02-48.

[3] R. J. Fontana and E. A. Richley, "Observations on low data rate, short pulse UWB systems," in Proceedings of the IEEE International Conference on Ultra-Wideband (ICUWB '07), pp. 334-338, September 2007.

[4] R. Jin, S. Halder, J. C. M. Hwang, and C. L. Law, "Tunable pulse generator for ultra-wideband applications," in Proceedings of the Asia Pacific Microwave Conference (APMC '09), pp. 17801783, December 2009.

[5] S. Halder, J. C. M. Hwang, G. A. Solomon, and G. Klein, "Order-of-magnitude improvement in microwave power capacity of InGaP/GaAs HBT under isothermal pulsed operation," IEEE Transactions on Electron Devices, vol. 53, no. 10, pp. 2634-2639, 2006.

[6] W. Liu, Handbook of III-V Heterojunction Bipolar Transistors, Wiley \& Sons, New York, NY, USA, 1998.

[7] HBT H02U-10 Technology, WIN Semiconductors, Taoyuan, Taiwan.

[8] S. Halder, R. Jin, J. C. M. Hwang, J. Lim, and S. Cheon, "Modeling and characterization of sub-nanosecond impulse response of high-voltage heterojunction bipolar transistors," in Proceedings of the IEEE MTT-S International Microwave Symposium (IMS '07), pp. 609-612, June 2007.

[9] R. Jin, C. Chen, S. Halder, W. R. Curtice, and J. C. M. Hwang, "Safe operating area of GaAs HBTs based on sub-nanosecond pulse characteristics," IEEE Transactions on Microwave Theory and Techniques, vol. 58, no. 12, Article ID 5620941, pp. 39964003, 2010.

[10] Agilent Technologies, Santa Rosa, Calif, USA.

[11] N. Deparis, C. Loyez, N. Rolland, and P. A. Rolland, "UWB in millimeter wave band with pulsed ILO," IEEE Transactions on Circuits and Systems II, vol. 55, no. 4, pp. 339-343, 2008.

[12] J. Han and C. Nguyen, "On the development of a compact subnanosecond tunable monocycle pulse transmitter for UWB applications," IEEE Transactions on Microwave Theory and Techniques, vol. 54, no. 1, pp. 285-293, 2006.

[13] J. Lai, Q. Hao, J. She, and Z. Feng, "A low cost trigger frequency alterable ultra-wide band ambipolar pulses generator," in Proceedings of the International Conference on Microwave and Milli meter Wave Technology (ICMMT'08), pp. 216-219, April 2008.

[14] J. Mrkvica, J. Jerabek, R. Sikl, and D. Dvorak, "The experimental UWB impulse radio link and jammer," in Proceedings of the 17th International Conference Radioelektro-nika Dig, April 2007.

[15] M. Miao and C. Nguyen, "On the development of an integrated CMOS-based UWB tunable-pulse transmit module," IEEE Transactions on Microwave Theory and Techniques, vol. 54, no. 10, Article ID 1705687, pp. 3681-3687, 2006.

[16] J. Lee, Y. J. Park, M. Kim, C. Yoon, J. Kim, and K. H. Kim, "System-on-package ultra-wideband transmitter using CMOS impulse generator," IEEE Transactions on Microwave Theory and Techniques, vol. 54, no. 4, pp. 1667-1674, 2006.
[17] G. Lim, Y. Zheng, W. Yeoh, and Y. Lian, "A novel low power UWB transmitter IC," in Proceedings of the IEEE Radio Freq-uency Integrated Circuits Symposium, pp. 1-4, June 2006.

[18] N. Sasaki, M. Fukuda, K. Kimoto, and T. Kikkawa, "CMOS UWB transmitter and receiver with silicon integrated antennas for inter-chip wireless interconnection," in Proceedings of the IEEE Radio and Wireless Symposium Dig (RWS '08), pp. 795-798, January 2008.

[19] B. Xin, X. Wang, A. Wang, H. Chen, and B. Zhao, "A programmable $1.8 \mathrm{pj} /$ pulse gaussian pulse generator for impulse UWB transceiver in $90 \mathrm{~nm}$ CMOS," in Proceedings of the IEEE Radio and Wireless Symposium (RWS '09), pp. 498-501, January 2009.

[20] F. Zito, D. Pepe, and D. Zito, "UWB CMOS monocycle pulse generator," IEEE Transactions on Circuits and Systems I, vol. 57, no. 10, Article ID 5467176, pp. 2654-2664, 2010.

[21] S. Bagga, A. Vorobyov, S. Haddad, A. Yarovoy, W. Serdijn, and J. Long, "Codesign of an impulse generator and miniaturized antennas for IR-UWB," IEEE Transactions on Microwave Theo-ry and Techniques, vol. 54, no. 4, pp. 1656-1666, 2006.

[22] L. H. Camnitz and N. Moll, "An analysis of the cutofffrequency behavior of microwave heterostructure bipolar transistor," in Compound Semiconductor Transistors-Physics and Technology, pp. 21-45, IEEE Press, New York, NY, USA, 1993.

[23] Cadence Design Systems, San Jose, Calif, USA.

[24] C. J. Wei, J. C. M. Hwang, W. J. Ho, and J. Aiden Higgins, "Large-signal modeling of self-heating, collector transit-time, and RF breakdown effects in power HBTs," IEEE Transactions on Microwave Theory and Techniques, vol. 44, no. 12, pp. 2641-2647, 1996. 

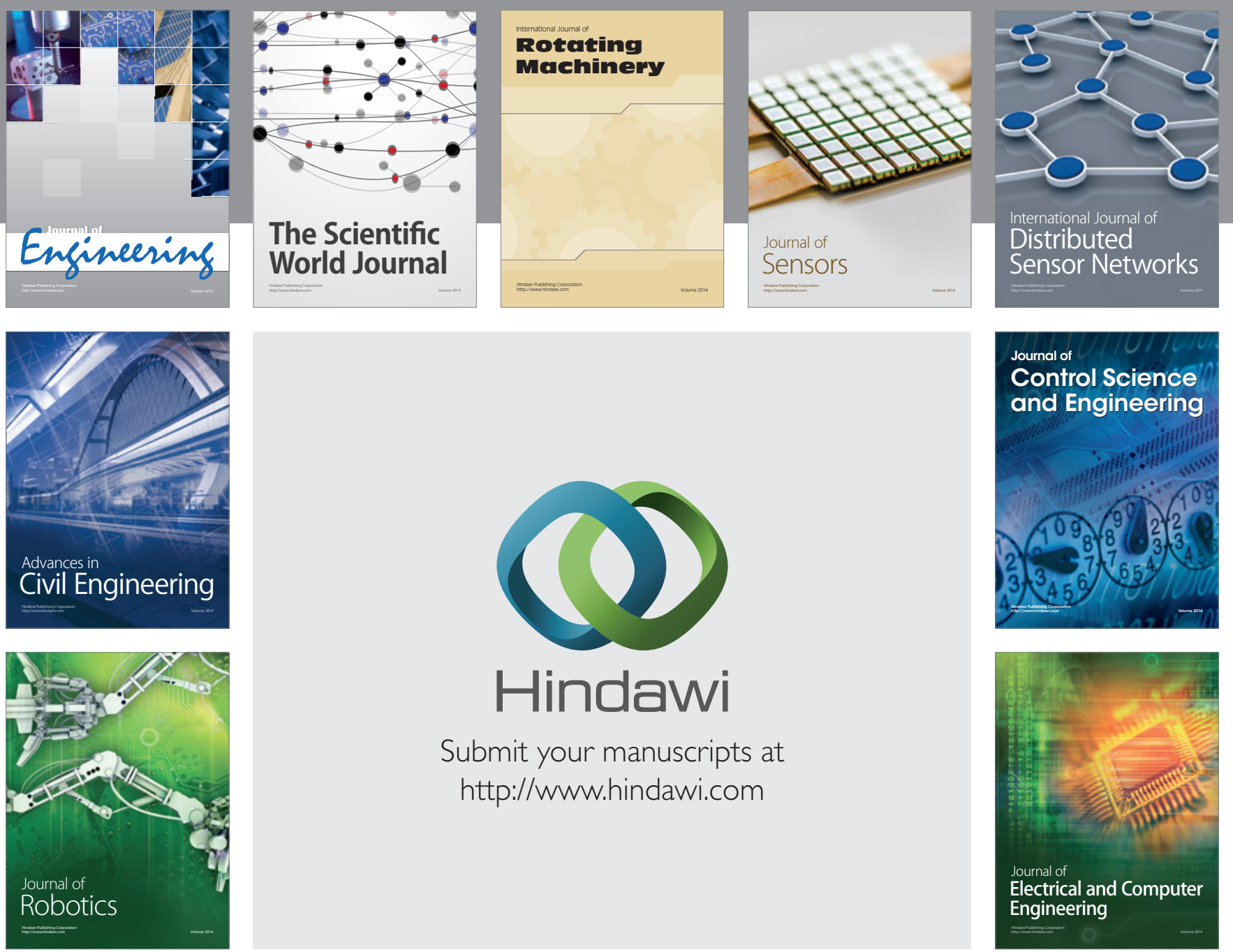

Submit your manuscripts at

http://www.hindawi.com
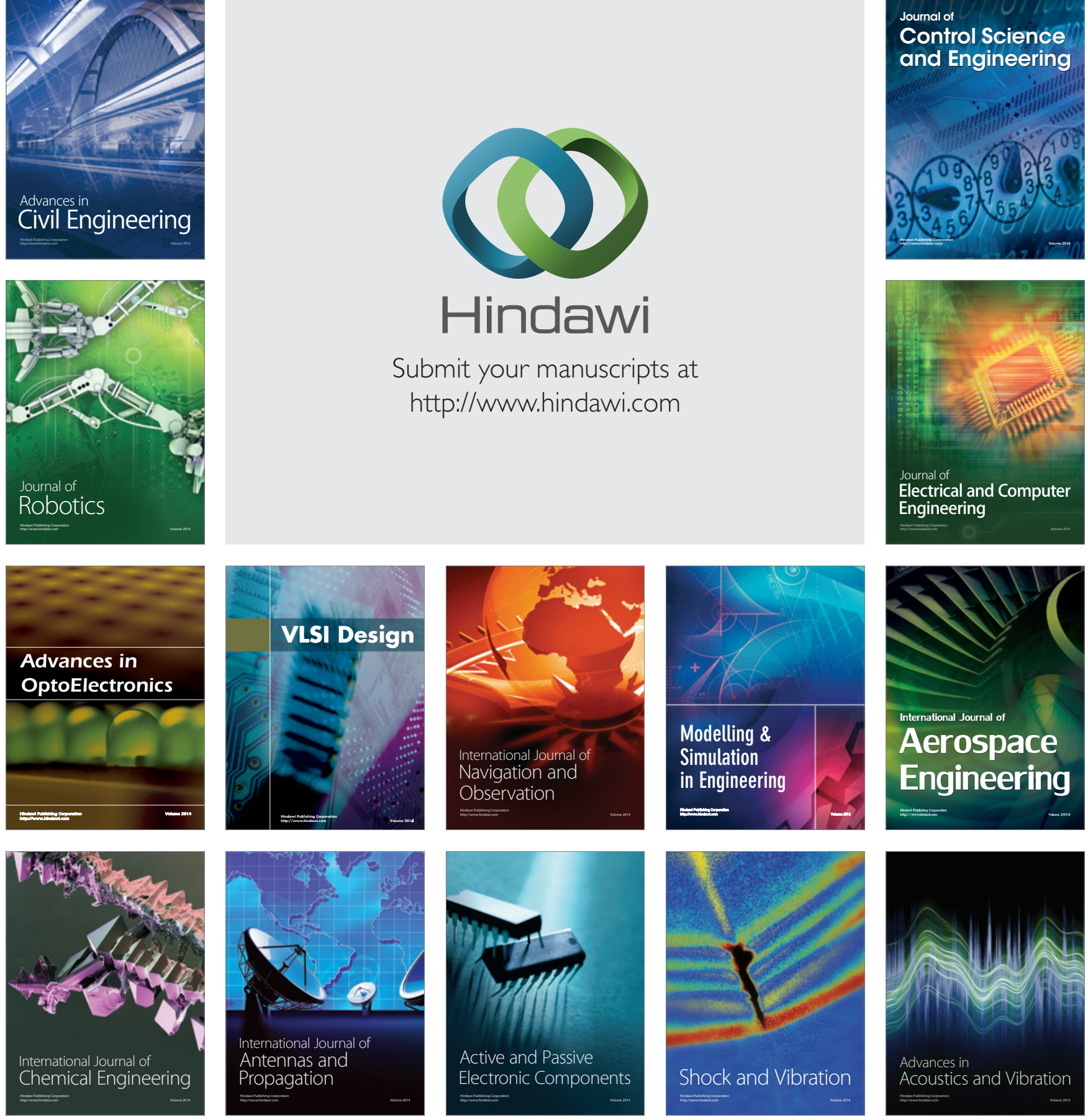